\title{
Effect of light Intensity and photoperiod on growth of Chlorella pyrenoidosa and $\mathrm{CO}_{2}$ Biofixation
}

\author{
Teuku Johar Gunawan ${ }^{1, *}$, Yusni Ikhwan ${ }^{2}$, Fajar Restuhadi ${ }^{3}$, and Usman Pato ${ }^{3}$ \\ ${ }^{1}$ Post graduate program of Environmental Science, Universitas Riau,Pekanbaru, Riau, Indonesia \\ ${ }^{2}$ Post graduate program of Environmental Science, Universitas Riau,Pekanbaru, Riau, Indonesia \\ ${ }^{3}$ Faculty of Agriculture, Univers itas Riau, Pekanbaru, Riau, Indonesia
}

\begin{abstract}
Microalgae have been viewed as one of potential solution for $\mathrm{CO}_{2}$ biofixation or $\mathrm{CO}_{2}$ sequestration. However, many factors need to be evaluated to support development of $\mathrm{CO}_{2}$ biofixation. One important environmental factor for the growth of micro algae is related with light requirement. The aim of this study was to evaluate the effect of light intensity and photoperiod on growth of Chlorellapyrenoidosa (C.pyrenoidosa) and $\mathrm{CO}_{2}$ biofixation. Experiments were carried out in $1000 \mathrm{~mL}$ semi batch photo bioreactors, purged continuously with air $\left(0.034 \% \mathrm{CO}_{2}\right)$. An Experiment of Factorial Design was employed in which the light intensity was evaluated 4 level at 2000, 4000, 6000 and 8000 lux with 3 level of photo period at L/D (light/dark) 8 hours/16 hours; L/D 12 hours/12 hours and L/D 16 hours $/ 8$ hours. The result indicated that both light intensity and photo period had significant effect $(\mathrm{p}<$ 0.05 ) on growth of $C$. pyrenoidosa. However, the photo period showed stronger effect relative to light intensity on growth of C.pyrenoidosa within the range reviewed. The interaction between the two factors was indicative but statistically not significant. Best growth profile sustained at combination of L/D 16 hours/ 8 hours of photoperiod and light intensity of 8000 lux with the highest average biomass observed at $0.516 \pm 0.069 \mathrm{gr} / \mathrm{L}$. An increase in $\mathrm{CO}_{2}$ biofixation rate of around 2 times was also observed between highest setting (8000 lux; L/D 16/12 hours) relative to that of lowest setting (2000 lux; L/D 8/12 hours).
\end{abstract}

\section{Introduction}

The global warming has become an attention to the international community and an important challenge[1].This global warming is due to an increase of several gases called green house gas. One of the contributing gases is carbon dioxide released by human activities such as the gas coming out from industrial and transportation sectors. The result of global warming is an increase in earth temperature.

The negative implication of global warming to human has been acknowledged such as a negative effect to human health [2] as well as to global environment in term of unusual weather and storm, and impact to the plant and animals [3]. The environmental impact of global warming has triggered efforts to reduce emission from the green house gas[4], [5], [6].

Carbon dioxide $\left(\mathrm{CO}_{2}\right)$ is one of important component of green house gas. Simulation on global and regional scenarios estimated that the carbon resulted from usage of fossil fuel (this includes gas and crude oil) will still be main source of green house gas emission in the next century. It also has estimated that in year 2100 carbon dioxide may reach to the level of 70 to $80 \%$ of total emission of green house gas [7]. Nowadays, around $40 \%$ of emission of $\mathrm{CO}_{2}$ is resulted from power generation fuelled by fossil fuel including gas, crude oil and diesel [4].

Many non biological methods which use physical and chemical process have been proposed to sequester carbon dioxide (carbon fixation or carbon capture) by researchers. Example of those methods are chemical absorption using Amine called Amine Absorption, adsorption using solid in example Zeolites or using Activated Carbon, physical process using membrane polymer and the use of cryogenic or very low temperature process to capture carbon [4],[8],[9]. Part of the method is also the idea to capture carbon and then to store it under (sub surface storage),[10], however another researcher reported the environmental impact of the sub surface storage mentioned above [11].

Biological method is another way to sequester carbon dioxide. Microalgae are viewed as one of potential solution for $\mathrm{CO}_{2}$ sequestration or $\mathrm{CO}_{2}$ biofixation beside major potential for biodiesel development which has better fuel characteristic [12], and has high lipid content [13]. Microalgae can also be cultivated along the year, have better sun light utilization and higher yield relative to terrestrial plants and advantage to be used as one method to sequester $\mathrm{CO}_{2}[14]$.

However many factors need to be evaluated to support development of $\mathrm{CO}_{2}$ Biofixation. One of

*Corresponding author: tig@consultant.com 
important environmental factor for the growth of microalgae is related with light requirements. Various reports were available on effect of different light intensity but limited report on effect of photoperiod to different strain of algae. One good paper reported the effect photoperiod on Apanoteche microscopic Nageli but at a single fixed light intensity of $150 \mu \mathrm{mol} / \mathrm{m}^{2} . \mathrm{s}$ through-out the experiment[15].

There was opportunity in term of factors evaluation to different strains especially on Chlorella pyrenoidosa as data available evaluating both factors was limited. Therefore the aim of our study was to evaluate the effect of light intensity and photoperiod on growth of Chlorella pyrenoidosa (C.pyrenoidosa) and $\mathrm{CO}_{2}$ biofixation.

Our approach is to evaluate both photoperiod and light intensity using the factorial design to see the main effects of both factorsand their significance on growth of C.pyrenoidosa and to see if there is a factor interaction. This approach is difference from traditional approach of one factor at a time evaluation.

The result of this study has contribution to provide a baseline for Chlorella pyrenoidosa in term of light regime requirements of both photoperiod and light intensity when purged by air with $\mathrm{CO}_{2} \quad 0.034 \%$. The knowledge has important implication for further cultivation application using flue gas as well as optimization schemes and economic valuation later on.

\section{Materials and Methods}

\subsection{Microalgae Strain and Medium}

The culture of Chlorella pyrenoidosa used in this study was from Jepara region, Java, Indonesia. Initial incubation was conducted in room temperature between $27-31{ }^{\circ} \mathrm{C}$, exposed to continuous lighting for 24 hours with light intensity of 2000 lux. Initial culture was grown in several photo bioreactor of 25 litres volume to provide sufficient volume for experimental runs.

Medium for growth used in the experiment was analternate novel medium fertilizer complex (G15Deptan) with composition of $5 \% \mathrm{NO}_{3}, 18 \% \mathrm{~K}_{2} \mathrm{O}$, $3 \% \mathrm{MgO}, 8 \% \mathrm{~S}, 0.35 \% \mathrm{Fe}, 0.02 \% \mathrm{Mn}, 0.02 \% \mathrm{Zn}$, and $0.015 \%$ Boron.

\subsection{Experimental set up, design and analysis}

The photo bioreactor used for the experiment were made of glass (Schot, German) with volume of $1000 \mathrm{~mL}$, diameter of $9.5 \mathrm{~cm}$. Total of 24 reactors were used for all experimental runs as per design.

Source of light was LED (light emitting diode) with total of 24 unit of 10 Watt LED (Hannoc, Indonesia) for each of reactor run. LED was position at adjustable distance to provide light intensity of 2000, 4000, 6000 and 8000 lux at the surface of the reactors measured with lux meter (LX-101A, Taiwan).Cycle of photoperiod was adjusted for 3 levels at L/D 8 hours of lighting and 16 hours of dark period, L/D 12 hours of lighting and 12 hours of dark period, and L/D 16 hours of lighting and 8 hours of dark period. Time was set for beginning and end of lighting accordingly for those 3 levels. A partition was put surrounding each reactor and an aluminum foil were used to cover top portion of the partition to isolate light from specific LED source to its associated single reactor.

Small air compressors were used to feed air continuously into each reactor. The objective is to supply $\mathrm{CO}_{2}$ (contains $\mathrm{CO}_{2}$ approximately $0,034 \%$ ) to be used for microalgae but also to help fluid mixing in the reactor. Overall configuration is shown in Figure 1.
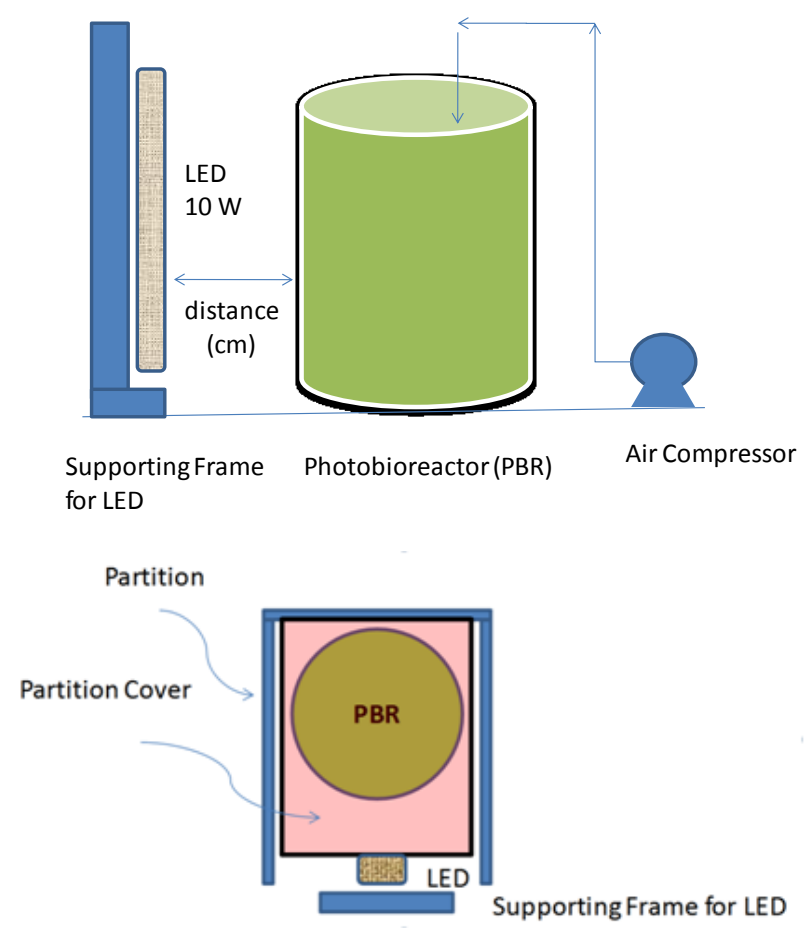

Fig. 1. Experimental set up and the top view of it.

Experiment was conducted semi batch. Microalgae fed into the reactor at the beginning of experiment and air was purged into the reactor continuously.

Design of experiment (DOE) used factorial design with 4 levels of light intensity (2000, 4000, 6000 and 8000 lux) and 3 levels of photoperiod (8, 12 and 16 hours of lighting and associated dark period accordingly). There were 24 runs out of 12 light intensity and photoperiod combinations including replicate.

Analysis of variance (ANOVA) was employed to see the significance of the main effect and interaction of factors involved. Tukey Post hoc Method wa sused for pair wise comparisons of means at $95 \%$ confidence level. Statistical package (Minitab-16) was used to help statistical analysis.

\subsection{Growth measurement}

Growth of the algae was determined daily by measuring optical density at wavelength of $680 \mathrm{~nm}$ (nano meter) using a spectrophotometer (Genesys 20, Thermo Scientific, USA). Prior to experimental run, curve of absorption spectrum of Chlorella pyrenoidosa was 
plotted at various wavelengths to determine the wavelength that will be used in the experiments. The wavelength of $680 \mathrm{~nm}$ was used as base for optical density measurement during the experiment.

\subsection{Dry weight determination}

Proxy method was used to get correlation between optical density and dry weight of biomass is used to determine dry weight of biomass in gram per litre $(\mathrm{gr} / \mathrm{L})$ when measured optical density data is available.

An amount of known volume of culture was taken into several Erlenmeyer flasks and then diluted to different ratio to provide range of optical density readings. Liquid was then transferred into test tubes $(10$ $\mathrm{mL}$ ), spinned for 60 minutes using centrifuge (Gemmy, Taiwan), filtered with filter paper (Whatman, German) and naturally dried in room temperature for 50 hours. Dry weight of biomass was determined gravimetrically using analytical balance (Kenko, Japan).

The resulted correlation given in Equation (1) is then used as a calibration curve to calculate the biomass dry weight in gram/litre based on the measured optical density data from experimental run.

$y=1.227 . x+0.126\left(\mathrm{R}^{2}=0.982\right)$

Where $y$ is the dry weight in $\mathrm{gr} / \mathrm{L}, x$ is the measured optical density or absorbance from the experiment.

\subsection{Biomass productivity and growth kinetic}

Biomass productivity is an increase in biomass per unit of time and calculated as per Equation (2) and the biomass productivity maximum was calculated by Equation (2).

$P=\left(X_{i}-X_{i-1}\right) /\left(t_{i}-t_{i-1}\right)$

Where $P$ is daily productivity, $X_{i}$ is the biomass at time $t_{i}, X_{i-1}$ is the biomass at time $\mathrm{t}_{\mathrm{i}-1}$.

Specific growth rate of microalgae was determined using the equation (3).

$\mu=\left[\log _{\mathrm{e}}\left(X_{t} / X_{t-1}\right)\right] / \triangle t$

Where $\mu$ is the specific growth rate (unit of $1 /$ day), $X i$ is the biomass at time $t i$ and $X i$ is the biomass at initial and $\Delta t$ is elapse time in reviewing $\mu$. The specific growth rate was calculated on the growth phase of the C.pyrenoidosa.

\subsection{Rate of $\mathrm{CO}_{2}$ biofixation}

The rate of $\mathrm{CO} 2$ biofixation was calculated as follows:

$$
R C O 2=C \cdot P .\left(\mathrm{MCO}_{2} / \mathrm{MC}\right)
$$

$R \mathrm{CO}_{2}$ is the rate of $\mathrm{CO}_{2}$ biofixation. $\mathrm{C}$ is the carbon content of biomass. $P$ is the productivity. $\mathrm{M} \mathrm{CO}_{2}$ is the molar mass of carbon dioxide and $\mathrm{MC}$ is the molar mass of carbon. Typical carbon content of biomass ranges from $41-51 \%$ of weight of biomass[16]. In our analysis the approach is to use $50 \%$ average of carbon content of biomass in Equation (4) following the average carbon content ( $\%$ weight) resulted from elemental analysis across several $\mathrm{CO}_{2}$ concentration on C.pyrenoidosa[17].

\section{Results and discussion}

Light is required for photosynthesis process of microalgae. Light intensity and photoperiod is an important part that not only can influence the growth of microalgae but in turn influence also the rate of $\mathrm{CO}_{2}$ biofixation.

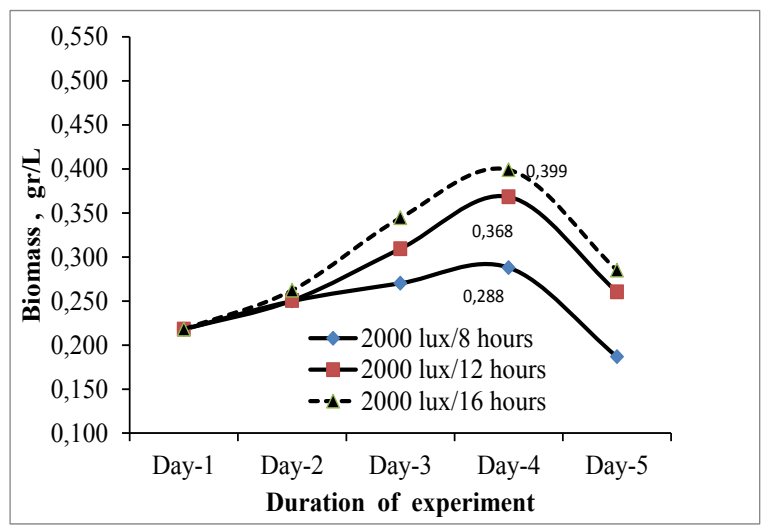

Fig. 2. Growth profile of C.pyrenoidosa at 2000 lux

\subsection{Growth profile and kinetics}

The growth profiles of Chlorella pyrenoidosa are presented as a plot of biomass versus time (Fig 2 to Fig 4). The growth profiles follow a general pattern of growth curve for a typical batch cultures phases from initial, logarithmic, declining growth, stationary and death phase, although clear distinction between phases differs from each condition in the experiment.

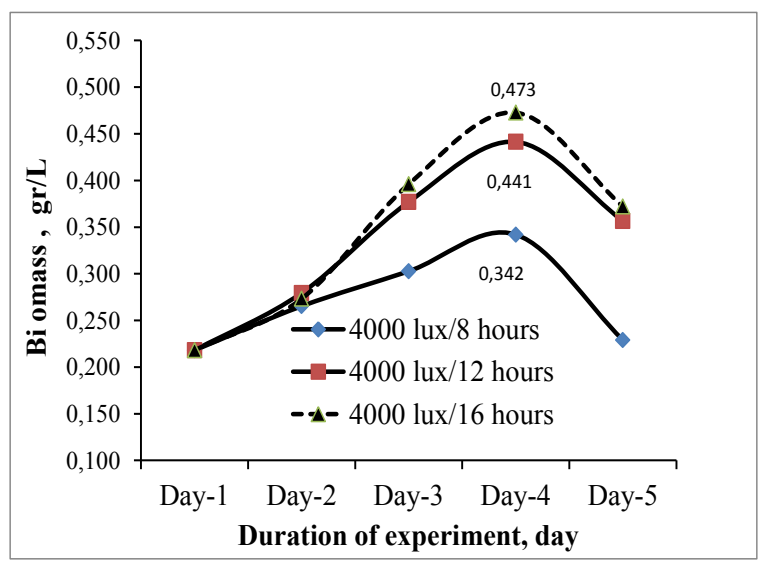

Fig. 3. Growth profile of C.pyrenoidosa at 4000 lux

The initial or lag phase were almost unnoticeable indicating high adaptability to the new growth medium 
after inoculums transfer to the reactors. The batch cultures reached the highest point on day 4 of cultivation and then entered the death phase for all condition as nutrient available for growth diminished, except the culture with light intensity of 8000 lux and 16 hours photoperiod that still sustained until day 6 of cultivation with highest biomass at $0.516 \pm 0.069 \mathrm{gr} / \mathrm{L}$ before entering death phase afterward.
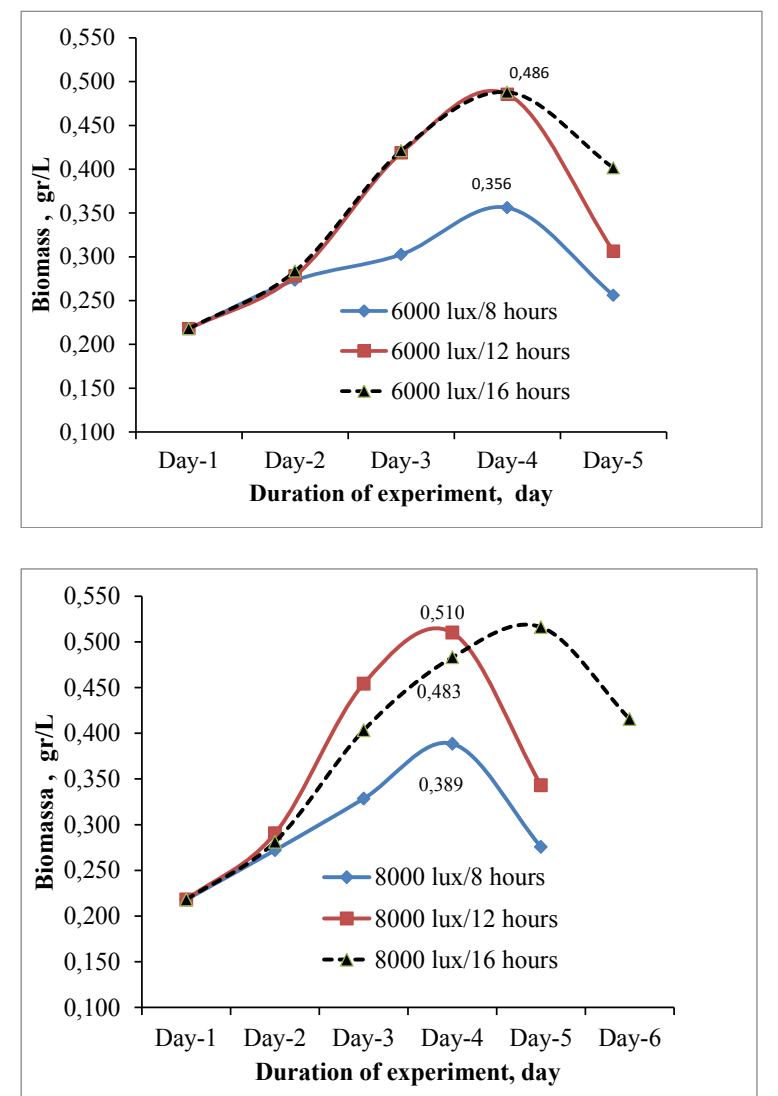

Fig. 4. Growth profile of C. Pyrenoidosa at 6000 and 8000 lux

The kinetic growth was reviewed at the logarithmic phase. The highest specific growth, $\mu\left(\mathrm{d}^{-1)}\right.$ of $C$. Pyrenoidosa was 0.447 at 8000 lux and 12 hours of photoperiod which was 5.7 times higher than the specific growth rate at 2000 lux and 8 hours of photoperiod and 1.2 times higher than specific growth at 8000 lux and 16 hours of photoperiod (Fig.5).

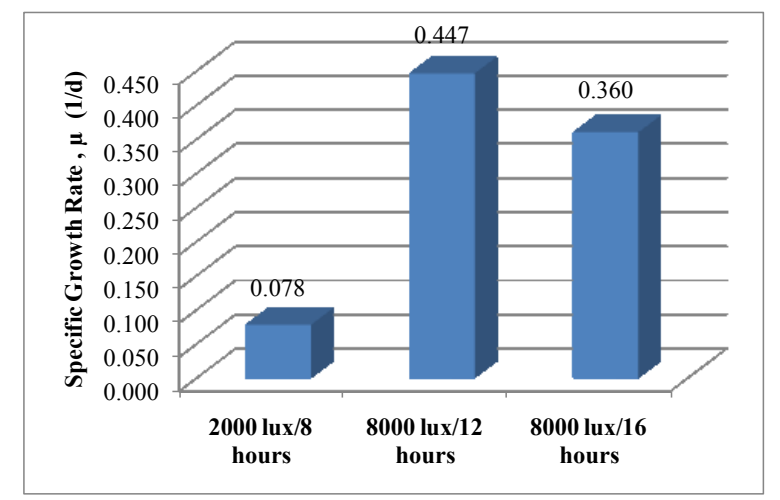

Fig. 5.Specific growth rate of C.pyrenoidosa between 2000 lux and 8000 lux at 12 and 16 hours of photoperiod.
Other researcher reported specific growth rate of several Chlorella strains at various conditions. It was reported that specific growth of Chlorella sorokiniana was $0.4 \mathrm{~d}^{-1}$; Chlorellavulgaris under $4 \% \mathrm{CO}_{2}$ and $8 \%$ $\mathrm{CO}_{2}$ were at the $0.41 \mathrm{~d}^{-1}$ and $0.51 \mathrm{~d}^{-1}$ respectively. Nanochloropsisgaditana, another strain was reported also at $4 \%$ of $\mathrm{CO}_{2}$ and $8 \%$ of $\mathrm{CO}_{2}$ with specific growth value of $0.44 \mathrm{~d}^{-1}$ and $0.45 \mathrm{~d}^{-1}[16]$.

All of those reported values are in the same order with the result of this study for Chlorella pyrenoidosa with specific growth rate of $0.45 \mathrm{~d}^{-1}$ (when subjected to air with around $0.034 \% \mathrm{CO}_{2}$, light intensity of 8000 lux and photoperiod of 12 hour) and growth rate of $0.36 \mathrm{~d}^{-}$ ${ }^{1}$ when subjected to 8000 lux but with photoperiod of 16 hours. Other reports had used $\mathrm{CO}_{2}$ while C.pyrenoidosa in our study used air nevertheless the specific growth is comparable showing the potential of C.pyrenoidosa to be cultivated to biofixed $\mathrm{CO}_{2}$.

\subsection{Factors main affect and interaction}

We observed that the biomass increases with the increase of light intensity. The similar trend was also observed with the increase of photoperiod. However the increase of biomass with increase of light intensity appeared to be more gradual than an increase of biomass due to an increase of photoperiod. The value of biomass reached0.399 gr/L at the 16 hours of photoperiod with light intensity of 2000 lux (Fig.2), while the value reached $0.473 \mathrm{gr} / \mathrm{L}$ with the same photoperiod but at 4000 lux light intensity (Fig.3). That was an increase of $18.5 \%$.

There was an increase of $27.8 \%$ and $38.5 \%$ of biomass if we move from photoperiod of 8 hours to 12 hours and from 8 hours to 16 hours respectively within the same light intensity of 2000 lux.

Similar trend was observed at 4000 lux intensity with the increase of biomass of $28.9 \%$ and $38.3 \%$, if we move from photoperiod of 8 hours to 12 hours and from 8 hours to 16 hours respectively.

The biomass differences at light intensity of 6000 lux and 8000 lux (Fig.4) also follow the same fashion when comparing value of biomass response for photoperiod 8 hours to 12 hours and 8 hours to 16 hours respectively. However, the percent increase of biomass was lower at 8000 lux compared to 6000 lux for the same duration of photoperiod 8 hours to 16 hours $(32.6 \%$ and $36.5 \%$ respectively).

In addition, the same percent increase was observed between 8 hours to 12 hours and 8 hours to 16 hours photoperiod $(36.5 \%)$.

The analysis of main effect of factors of light intensity and photoperiod revealed actual influence of factors to mean of response. The response of biomass had steeper increase from light intensity of 2000 lux to 4000 lux and from 8 hours to 12 hours photoperiod (Fig.7).

Mean of biomass gradually increased from light intensity of 4000 lux to 8000 lux and from 12 hours to 16 hours of photoperiod. 
Interaction between light intensity and photoperiod was indicative from the result above. Analysis of interaction between the factors shows that although increasing photoperiod will certainly increase the mean of response as indicated in the main effect; however the response was dependent on the level of light intensity to some degree. The interaction was less obvious from 8 hours to 12 hours of photoperiod relatively to 12 hours to 16 hours of photoperiod across the range of light intensity (Fig.8).

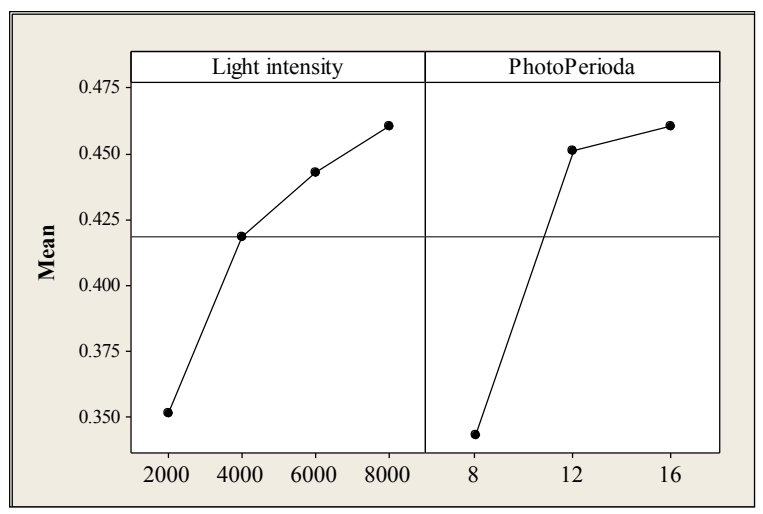

Fig. 7.Main effect plot for biomass (light intensity in lux and photoperiod in hours) of C.pyrenoidosa

Results above indicated that the photoperiod had greater affect to biomass relatively than the changes in light intensity within the range reviewed in this experiment. One good report on the effect of photoperiod, although evaluated to different microalgae strain - Apanoteche microscopic Nageli and used only a single fixed light intensity of $150 \mu \mathrm{mol} / \mathrm{m}^{2}$.s through-out the experiment -, had observed that the photoperiod was a determinant factor and linear reduction in response was also observed with reduction of photoperiod.[15]. Our study confirmed the observation as well.

In order to examine the statistical significance of factor affects, the analysis of variance (ANOVA) was employed.

Analysis of variance result (Table.1) confirmed that the two factors of light intensity and photoperiod were statistically significant $(p<0.05)$, with $p$ value of light intensity factor greater than photoperiod although still less than 0.05 , confirming the visual observation of stronger effect of photoperiod relative to light intensity.

Interaction term between light intensity and photoperiod although indicative from the plot was statistically not significant $(p=0.885$ which is $>0.05)$. In order to see which of combination of light intensity and photoperiod were significant out of 12 experimental runs, we employed Tukey pairwise test at 95\% confidence.

TukeyTest provided difference of means of biomass response among level of light intensity and photoperiod. The difference response of biomass between light intensity of 2000 lux to all other light intensity from 4000 lux, 6000 lux and 8000 lux were statistically significant (all value of $p<0.05$ ). The $p$ value for difference biomass response between 4000 lux to 6000 lux and 8000 lux ligh intensity were not statistically significant ( $p=0.618$ and 0.269 respectively). The difference biomass reponse between 6000 lux and 8000 lux was also not significant ( $p=0.8545$ ).

The grouping information using the same method at $95 \%$ confidence showed that 16 hours and 12 hours of photoperiod were within the same group and 8 hours of photoperiod were in another difference group in term of differencesin responses (Table.2)

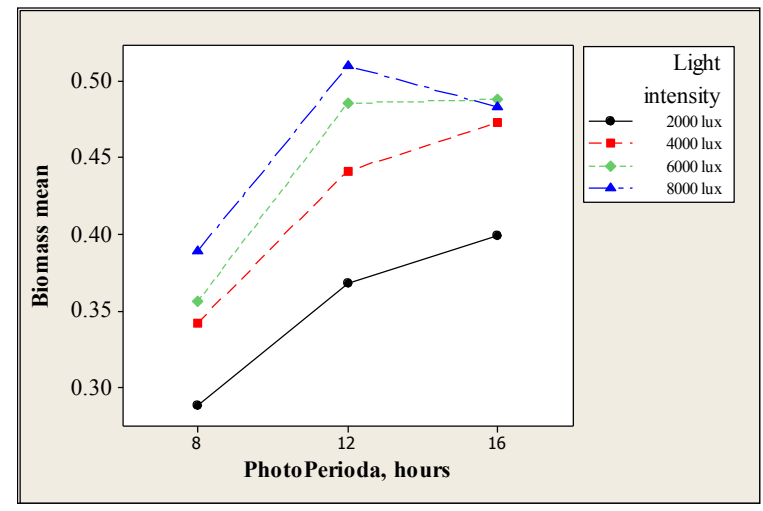

Fig. 8. Interaction plot for biomass of C.pyrenoidosa

Table 1.ANOVA from Minitab output.

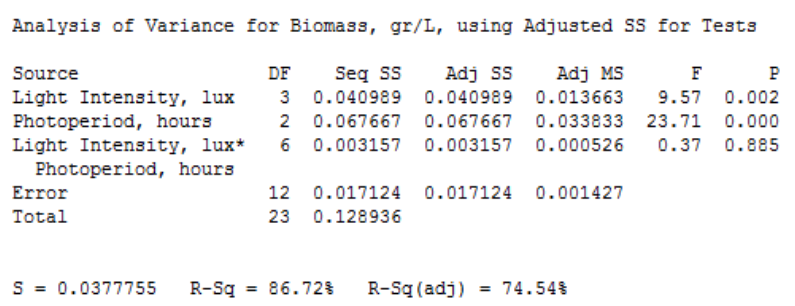

Table 2. Tukey pairwise comparison of means of biomass to photoperiod (L/D) - Minitab output.

Grouping Information Using Tukey Method and 95.0\% Confidence

$\begin{array}{lrrl}\text { Photoperiod, } & & & \\ \text { hours } & \text { N } & \text { Mean } & \text { Grouping } \\ 16 & 8 & 0.5 & \text { A } \\ 12 & 8 & 0.5 & \text { A } \\ 8 & 8 & 0.3 & \text { B }\end{array}$

Means that do not share a letter are significantly different.

The Tukey Test at 95\% confidence confirmed that the difference in response were statistically significant from 8 hours of L/D to 12 hours and 16 hours of $\mathrm{L} / \mathrm{D}(p$ $=0.0003$ and $p=0.0001$ respectively, all $p$ were $<0.05$ ), but L/D of 12 hours to L/D 16 hours was not statistically significant $(p=0.8749>0.05)$.

The practical meaning of the comparison above is that there is a threshold value of light intensity and photoperiod setting for cultivation of C.pyrenoidosa. Light intensity of 2000 lux is too low for cultivation as it 
did not provide sufficient energy to achieve best growth. The 8 hours of photoperiod or L/D of 8 hours of light exposure and 16 hours associated dark hours was not sufficient in term of timing to accumulate light energy necessary for the growth.

On the other hand, 12 hours of photoperiod with L/D 12 hours of light and 12 hours dark was sufficient for growth provided the appropriate light intensity is available. The additional 4 hours of light exposure at L/D 16 hours light /8 hours dark did not significantly different than the 12 hours of light exposure in term of biomass response; however. It differed in term of sustainability prior to entering the death phase.

The comparison indicated also that there was more flexibility in term of light intensity choices beyond the 4000 lux to 8000 lux provided the photoperiod is within the range of 12 hours of light exposure to 16 hours of it, although results from this study suggested that best region is close to 8000 lux with 12 hours or 16 hours of photoperiod if we neglect the possibility of curvature (where maximum point may be located) between those two photoperiods.

\subsection{Rate of $\mathrm{CO}_{2}$ biofixation of C.pyrenoidosa}

$\mathrm{CO}_{2}$ biofixation rate was determined based on calculated biomass daily productivity using Equation (2). The average rate of $\mathrm{CO}_{2}$ biofixation of C.pyrenoidosa at 12 factorial combination of photoperiod and light intensity ranges from $0.082 \pm 0.03 \mathrm{gr} / \mathrm{L}^{-1} \cdot \mathrm{d}^{-1}$ at 2000 lux and 8 hours of photoperiod to $0.267 \pm 0.02 \mathrm{gr} / \mathrm{L}^{-1} \cdot \mathrm{d}^{-1}$. That was an increased of about 2 times or the ratio of almost 3 folds value of the rate from the low setting of light intensity and photoperiod to the higher setting of them (Table. 3).

Table 3. Rate of $\mathrm{CO}_{2}$ biofixation by C.pyrenoidosa at 12 experimental conditions of light intens ity and photoperiod.

\begin{tabular}{|c|c|c|c|}
\hline \multirow{2}{*}{$\begin{array}{c}\text { Experimental condition } \\
\text { (light intensity } \& \\
\text { photoperiod) }\end{array}$} & \multicolumn{3}{|c|}{$\begin{array}{c}\text { CO2 Biofixation, }\left(\mathrm{gr} / \mathrm{L}^{-1} \cdot \mathrm{d}^{-1}\right) \\
\text { means } \pm \mathrm{sd}^{*}\end{array}$} \\
\hline & 0.082 & \pm & 0.03 \\
\hline 2000 lux/12 hours & 0.132 & \pm & 0.04 \\
\hline 2000 lux/16 hours & 0.165 & \pm & 0.03 \\
\hline 4000 lux/8 hours & 0.133 & \pm & 0.02 \\
\hline 4000 lux/12 hours & 0.211 & \pm & 0.01 \\
\hline 4000 lux/16 hours & 0.223 & \pm & 0.01 \\
\hline 6000 lux/8 hours & 0.152 & \pm & 0.01 \\
\hline 6000 lux/12 hours & 0.237 & \pm & 0.00 \\
\hline 6000 lux/16 hours & 0.245 & \pm & 0.03 \\
\hline 8000 lux/8 hours & 0.170 & \pm & 0.01 \\
\hline 8000 lux/12 hours & 0.267 & \pm & 0.02 \\
\hline 8000 lux/16 hours & 0.239 & \pm & 0.06 \\
\hline
\end{tabular}

of light intensity and photoperiod for cultivation from this study could provide comparable result of $\mathrm{CO}_{2}$ biofixation rate to other cultivation condition reported.

Our study indicated the important of length of exposure to light (photoperiod) and light intensity level selection to achieve desired result of $\mathrm{CO}_{2}$ biofixation out of microalgae cultivation. In general the result showed an increase of $\mathrm{CO}_{2}$ biofixation rate at the increasing combination of factors, however the highest average rate of $\mathrm{CO}_{2}$ biofixation was achieved at high setting of light intensity (8000 lux) and 12 hours of photoperiod not at the 16 hours photoperiod. Interaction plot of biomass also reveal the same phenomenon (Fig.8). This was due to higher interaction occurring at 8000 lux and 16 hours relative to lower light intensity and lower photoperiod. In addition, the phenomenon also may indicate the existence of curvature on the response which may happen between photoperiod of 12 hours to 16 hours. It means that the maximum response could be located between 12 hours to 16 hours of photoperiod.

Our study also indicated that to achieve the comparable rate of $\mathrm{CO}_{2}$ biofixation (purged with air), relatively lower light intensity range used in this experiment (2000 lux to 8000 lux) could suffice the light requirement for cultivation. Economic wise, this is a desirable aspect for mass cultivation.

The result provided practical implication in term of region of light intensity and length of light exposure to achieve best $\mathrm{CO}_{2}$ fixation rate within the range of review. Light intensity needs to be between 6000 lux and 8000 lux and photoperiod to be between 12 hours to 16 hours to be in the region of highest rate. The best route for higher rate of $\mathrm{CO}_{2}$ biofixation is diagonal upward to the right of contour plot (Fig.9).

However, we need to note that the best region in the contour plot $\mathrm{CO}_{2}$ biofixation rate mentioned above does not necessarily pointed out the actual maximum point that can be achieved by C.pyrenoidosa but it indicated regions of combination of photoperiod and light intensity that we can expect to be the best route within the range reviewed in this experiment.

The actual maximum point location could be located within the region but to pin point the actual maximum location, the optimization study will need to be performed later on due to the possibility of curvature between light intensity of 6000 and 8000 lux and photoperiod of 12 hours and 16 hours based on contour plot review.

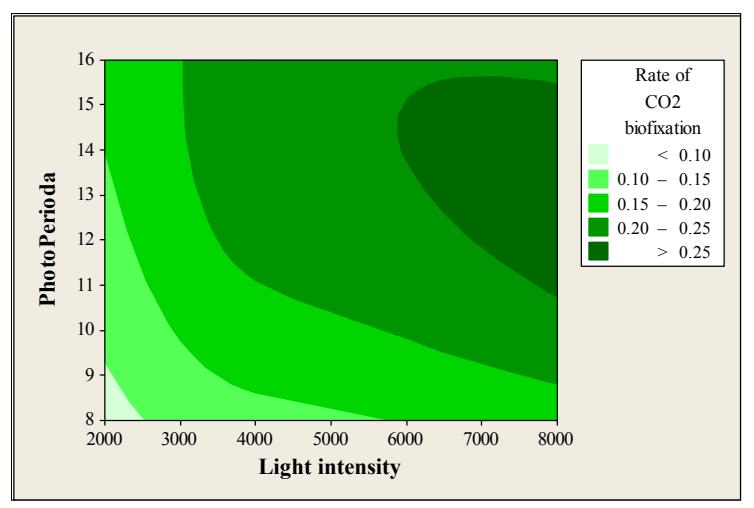


Fig. 9. Contour plot showing region of light intensity and photoperiod for $\mathrm{CO}_{2}$ biofixation of C.pyrenoidosa (Air with $\mathrm{CO}_{2}$ 0.034\%).

Therefore the result of this study is important baseline for further study on the optimization of C.pyrenoidosa.

Report(s) available on rate of $\mathrm{CO}_{2}$ biofixation of Chlorella pyrenoidosa is limited. The previous reported value of $\mathrm{CO}_{2}$ biofixation rate on the strain was available only with one level of light intensity used at 150 $\mu \mathrm{mol} /\left(\mathrm{s} . \mathrm{m}^{2}\right)$ and one photoperiod with $10 \% \mathrm{CO}_{2}$ and air [17], therefore to some extend could obscure the important of both effects of photoperiod and light intensity from those reports.

The rate of $\mathrm{CO}_{2}$ biofixation of C.pyrenoidosa from our study was comparable than the value previously reported by other researcher under different culture condition with $10 \% \mathrm{CO}_{2}$, whilethe result of this study showed around 1.7 to 2 times higher than the value reported by other research under the same condition with air. (Table.4).

Table 4.Several reported $\mathrm{CO}_{2}$ biofixation rate.

\begin{tabular}{|c|c|c|c|}
\hline Mikroalgae & $\mathrm{CO} 2 \%$ & $\begin{array}{c}\text { Rate of } \\
\mathrm{CO} 2 \\
\text { Biofixation } \\
(\mathrm{gr} / \mathrm{l} / \mathrm{d})\end{array}$ & Condition / References \\
\hline $\begin{array}{l}\text { C.pyrenoidosa } \\
\text { (Tang et al, } \\
\text { 2011) }\end{array}$ & $\mathrm{CO} 2,10 \%$ & 0,26 & $\begin{array}{l}180 \mu \mathrm{mol} /\left(\mathrm{s} . m^{2}\right) \sim \\
10,000 \mathrm{lux}, \mathrm{BG} 11 \\
{[17]}\end{array}$ \\
\hline $\begin{array}{l}\text { C.pyrenoidosa } \\
\text { (Tang et al, } \\
\text { 2011) }\end{array}$ & $\begin{array}{l}\mathrm{CO} 2,0.03 \\
\%\end{array}$ & 0,134 & $\begin{array}{l}180 \mu \mathrm{mol} /(\mathrm{s} . \mathrm{m} 2) \sim \\
10,000 \mathrm{lux}, \mathrm{BG} 11 \\
{[17]}\end{array}$ \\
\hline $\begin{array}{l}\text { C.pyrenoidosa } \\
\text { (this study, } \\
\text { 2017) }\end{array}$ & $\begin{array}{l}\text { Air, } \\
0.034 \% \\
\mathrm{CO} 2\end{array}$ & 0,239 & $\begin{array}{l}\text { average value at } \\
\text { condition of } 8000 \text { lux, } \\
16 \text { hours of } \\
\text { photoperioda, novel } \\
\text { fertizer complex } \\
\text { medium } \\
\end{array}$ \\
\hline $\begin{array}{l}\text { C.pyrenoidosa } \\
\text { (this study, } \\
\text { 2017) }\end{array}$ & $\begin{array}{l}\text { Air, } \\
0.034 \% \\
\mathrm{CO} 2\end{array}$ & 0,267 & $\begin{array}{l}\text { average value of } 8000 \\
\text { lux, } 12 \text { hours of } \\
\text { photoperioda, novel } \\
\text { fertilizer complex } \\
\text { medium }\end{array}$ \\
\hline
\end{tabular}

\section{Conclusions}

The light intensity and photoperiod as part of the light requirement were both statistically significant to the growth as well as the ability of C.pyrenoidosa to fix $\mathrm{CO}_{2}$. Photoperiod showed higher influence than light intensity to the growth and rate of $\mathrm{CO}_{2}$ biofixation.

It is important and economically desirable feature to be able to set suitable light regime for microalgae cultivation. The outcome of this study provided the best route of biomasss, specific growth rate and $\mathrm{CO}_{2}$ biofixation rate of C. Pyrenoidosa to be within region of
6000 lux to 8000 lux and between 12 hours and 16 hours of photoperiod (length of exposure to light).

The result can be used as a baseline for further evaluation of C.pyrenoidosa with other source of $\mathrm{CO}_{2}$ (i.e. flue gas), as well as optimization and economic valuation later on.

\section{References}

1. M. K. Lam, K. T. Lee, and A. R. Mohamed, Int. J. Greenh. Gas Control, 10, 456-469 (2012)

2. M. H. R. Anstey, Global. Health, vol. 9, no. 1, 4 (2013)

3. A. Yakub, P. Shrivastava, K. Krishnamurthi, S. N. Mudliar, S. Saravana, G. S. Kanade, S. K. Lokhande, and T. Chakrabarti, Bioresour. Technol., 138, 382-386 (2013)

4. Hadiyanto, Azimatun Nur, M.M.World Applied Sciences Journal, 31 (5), 959-967) (2014)

5. M. Meinshausen, N. Meinshausen, W. Hare, S. C. B. Raper, K. Frieler, R. Knutti, D. J. Frame, and M. R. Allen, Nature, 458, no.7242, 1158-1162 ( 2009)

6. J. Ball, Wall Str. J. East. Ed., 252, 70, A13 (2008)

7. T. Kram, T. Morita, K. Riahi, R. A. Roehrl, S. Van Rooijen, A. Sankovski, and B. De Vries, Technol. Forecast. Soc. Change, 63, no.2-3, 335371 (2000)

8. A. Hussain and M.-B. Hägg, J. Memb. Sci., 359, no. 1-2, 140-148 (2010)

9. M. J. Tuinier, M. van Sint Annaland, and J. a. M. Kuipers, Int. J. Greenh. Gas Control, 5, no. 4, 694-701 (2011)

10. J.-P. Nicot, S. Solano, J. Lu, P. Mickler, K. Romanak, C. Yang, and X. Zhang, Energy Procedia, 37, 4552-4559 (2013)

11. J. Koornneef, A. Ramírez, W. Turkenburg, and A. Faaij, Prog. Energy Combust. Sci., 38, no. 1, 6286 (2012)

12. Y. C. Sharma, B. Singh, and S. N. Upadhyay, Fuel, 87, no. 12, 2355-2373 (2008).

13. E. Suali and R. Sarbatly, Energy Rev, 16, no. 6, 4316-4342 (2012)

14. B. Wang, Y. Q. Li, N. Wu, and C. Q. Lan, Appl. Microbiol. Biotechnol., 79, 707-718 (2008)

15. E. Jacob-lopes, C. Henrique, G. Scoparo, L. Mara, and C. Ferreira, Chem. Eng. Process. Process Intensif., 48, 306-310 (2009)

16. M. Adamczyk and J. Lasek, App Biochem Biotech., 179, 1248-1261 (2016)

17. D. Tang, W. Han, P. Li, X. Miao, and J. Zhong, Bioresour. Technol., 102, no. 3, 3071-6 (2011) 